\title{
Paciente Roncador
}

Se denomina ronquido al sonido producido por la vibración de los tejidos blandos de la región de la faringe (el paladar blando y tal vez la úvula) debido al cambio de la configuración y las propiedades de la vía aérea superior que ocurre durante el sueño.

La mayoría de los pacientes que roncan no lo saben, sino por un tercero, quien suele ser el compañero/a de cama. Por lo tanto se entiende al ronquido como un fenómeno subjetivo, que depende de quien lo oye. Si bien ha tratado de ser definido y medido por estudios de sonido, no se han llegado a obtener parámetros universalmente aceptados.

Estudios poblacionales muestran que uno de cada cinco adultos de edad media ronca, el $24 \%$ son varones de edad media y el $15 \%$ son mujeres. Como la prevalencia del ronquido en la población es elevada, resulta un motivo frecuente de consulta en la práctica generai.

Algunos trabajos muestran relación entre la presencia de ronquido e hipertensión arterial diurna, enfermedad cerebrovascular y enfermedad coronaria. Pero muchos de estos estudios que encontraron asociación independiente entre la presencia de ronquido y los eventos vasculares no han incluído gran número de pacientes, no siempre utilizaron grupos adecuados de control y la presencia de múltiples confundidores como el sexo, la edad, el peso y el tabaquismo pudieron llevar a conclusiones inadecuadas. Por todo lo anterior, no se puede hablar de causalidad entre ronquido y enfermedad vascular, sino de posible asociación.

Durante el sueño, la presión negativa inspiratoria tiende a colapsar la vía aérea superior (VAS), lo cual es evitado por la acción de los músculos dilatadores de la faringe. Cualquier factor que tienda a disminuir el calibre de la VAS (en uno o más puntos) o altere la acción efectiva de los músculos dilatadores favorece la disminución de diámetro durante la inspiración. Dependiendo del grado de angostamiento producido, pueden verse tres fenómenos:

1) Que la reducción del calibre de la VAS no comprometa el flujo aéreo, pero sí lo tranforme en turbulento, generando una vibración en la VAS conocida como ronquido.

2) Que, a pesar del esfuerzo inspiratorio, exista una significativa caída del flujo aéreo sin llegar al cese del mismo (hipopnea obstructiva del sueño).

3) Que el colapso sea cada vez más significativo hasta producir el cese total del flujo aéreo a pesar del esfuerzo inspiratorio (apnea obstructiva del sueño). Los factores que se relacionan con los cuadros anteriores son: (Ver Esquema 1)

- El uso de sedantes-tranquilizantes, relajantes musculares y alcohol. - La obesidad. Cabe aclarar que el depósito de grasa perifaríngea es mayor en pacientes roncadores y/o con apnea obstructiya del sueño, que en los no roncadores de igual sexo, peso y edad.

- Tabaquismo.

- Alteraciones anatómicas en la VAS en diferentes puntos (hipertrofia amigdalina, obstrucción nasal y alteraciones craneofaciales, como la reducción de tamaño mandibular).

- El sexo masculino. Se han postulado como causas a las hormonas sexuales masculinas y a la configuración de la VAS en el varón, pero aứn no es clara la razón.

- La hipotonía de los músculos dilatadores de la faringe durante el sueño (principalmente la fase REM), hipótesis que ha sido cuestionada recientemente.

- Alteraciones que el ronquido podría producir crónicamente sobre el tejido de La VAS. Esquema 1: Fisiopatología

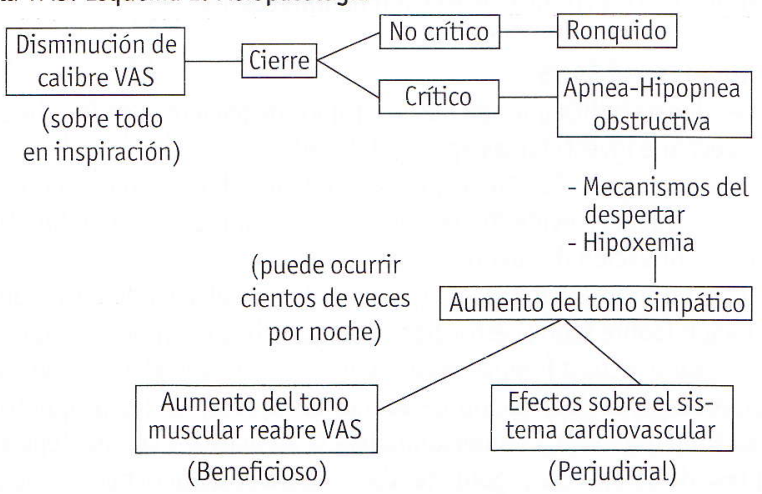

\section{¿Cómo se presenta el paciente roncador? :}

La mayoría de los pacientes roncadores son asintomáticos y no perciben el fenómeno durante el sueño, por lo que suelen enterarse mediante el compañero/a de cama, que suele ser quien muchas veces impulsa al paciente a la consulta. La principal manifestación del ronquido, es la disrupción del sueño de un tercero. El paciente roncador suele referir este problema dentro del examen de salud o como uno más de varios motivos de consulta. El ronquido puede darse durante la respiración nasal, bucal o combinada, aunque en el caso de la primera suele ser de menor intensidad.

Ante un paciente roncador debe ponerse un énfasis particular en la pesquisa de una somnolencia diurna excesiva cuya causa no sea evidente en el interrogatorio (ej. deprivación del sueño, consumo de benzodiacepinas, ingesta excesiva de alcohol, retención de $\mathrm{CO} 2$ en las excacerbaciones de la enfermedad obstructiva crónica), ya que esto conduce a la realización de estudios para el diagnóstico del sindrome de apnea-hipopnea ostructiva del sueño (SAHS) (ver desarrollo más adelante). Por la misma razón debe preguntarse al compañero de cama si ha percibido que el paciente detiene su respiración durante el ronquido. Debe consignarse si el paciente fuma, si consume alcohol o sedantes y si ha subido de peso.

El examen físico debe estar orientado fundamentalmente a la cabeza y al cuello. Debe examinarse la nariz en busca de obstrucción nasal o de desviación septal, las fauces en busca de alteraciones en la lengua, del paladar blando o de las amígdalas. Se debe evaluar el cuello para determinar si es corto $o$ ancho e inspeccionar el macizo facial en busca de alteraciones. No se debe olvidar, por la asociación antes discutida, el registro de tensión arterial y del peso.

\section{¿Qué exámenes complementarios deben realizarse?:}

El paciente asintomático:es decir el roncador sin somnolencia diurna, en quien no se han observado episodios de cese de la respiración, que no presenta alteraciones craneofaciales o de la VAS evidentes no requiere estudio adicional alguno. La evaluación polisomnográfica de los roncadores asintomáticos lleva a resultados normales en más del $80 \%$ de los casos, lo que muestra claramente su poca costo-efectividad.

En cambio cuando un paciente roncador presenta somnolencia diurna excesiva (de causa no explicable por otras razones) debe ser estudiado con una polisomnografía por el riesgo de apneas del sueño y más aún si el roncador con somnolencia diurna es obeso $\mathrm{y} / \mathrm{o}$ hipertenso o si el familiar ha observado episodios de cese de la respiración.

En quienes son candidatos a una cirugía nasal o faríngea por ronquido (ver tratamientos) puede considerarse la polisomnografía si reúnen otros factores de riesgo para apneas del sueño, ya que, de estar presente este cuadro, puede ofrecérseles un tratamiento no quirúrgico.

Respecto a los estudios de imágenes existen mediciones cefalométricas craneales tomográficas o por resonancia magnética. Sin embargo, éstas no predicen éxito quirúrgico en pacientes roncadores, tengan o no apneas del sueño.

\section{Tratamientos para el paciete roncador}

Tratamiento no quirúrgico. Medidas generales

1. Disminución del peso: la obesidad es uno de los factores más relacionados con el ronquido y/o las apneas del sueño. La disminución de peso puede hacer desaparecer o mejorar significativamente los episodios de ronquido, debiéndo, por lo tanto, enfatizarse este punto. No se puede predecir que reducción de peso es necesaria para lograr un beneficio respecto del ronquido, pero se sabe que no es indispensable lograr el peso ideal por tablas.

2. Evitar el alcohol y los sedantes, en especial, previamente a las horas de sueño. 3. Suspender el tabaquismo.

4. Sueño en decúbito lateral: algunos pacientes presentan menos episodios de ronquido o menor intensidad del mismo cuando duermen en decúbito lateral. Si bien este fenómeno no es cierto para todas las personas, ni hay datos claros al respecto, es una medida fácil de tomar. Hay quienes colocan pelotas de goma en la espalda de sus pijamas, para obligar al mantenimiento de esta posición durante el sueño.

5. Medicación nasal: esta medicación (en general tópica) se emplea con el fin de disminuir la resistencia nasal y promover la respiración por esta vía. 
No han demostrado un beneficio claro, pudiendo las de base oleosa (ej. fosfocolinamina) producir neumonía lipoidea, por lo tanto se desaconseja su uso.

6. Dilatadores nasales: los dilatadores nasales son aparatos que tienen por finalidad mantener permeabilidad nasal, pero hay pocos estudios para sacar conclusiones.

7. Aparatos de avance mandibular: son aparatos de aplicación oral, que desplazan el maxilar inferior hacia delante. Son confeccionados por odontólogos y/o técnicos dentales. Su utilidad está probada en las apneas del sueño (ver adelante) y podrían tener algún rol en el tratamiento de roncadores, debemos aguardar más conocimiento en este terreno.

\section{Tratamiento quirúrgico}

Cuando hay una causa anatómica evidente de obstrucción nasal o faríngea (ej. hipertrofia amigdalina, desviación del septum nasal, hipertrofia de las adenoides) puede considerarse la cirugía, aunque no puede predecirse con certeza el éxito del tratamiento. Sin embargo, es importante aclarar que en la mayoría de los pacientes que roncan no se encuentran alteraciones anatómicas pasibles de corrección quirúrgica.

La cirugía más estudiada en el caso de los roncadores asintomáticos es la uvulopalatofaringoplastia. La misma podría ser exitosa en un 75 a un $80 \%$ de los casos, aún en ausencia de alteraciones documentadas de la VAS. Debe advertirse que las series estudiadas son pequeñas y que el beneficio podría perderse con el paso del tiempo. Las cirugías realizadas mediante láser, carecen aún de resultados que las avalen.

Aunque el paciente parezca un claro candidato a la cirugía, primero debe hacerse énfasis en el cumplimiento de las medidas generales, sobre todo la disminución del peso en el caso de los pacientes obesos. Aquellos que han cumplido las medidas generales, pero que aún siguen roncando en forma no tolerable para su compañero/a de cama, puede ofrecérseles la realización de una uvulopalatofaringoplastia.

\section{Sindrome de Apnea-hipopnea del sueño}

El cese del flujo aéreo (apnea), así como la disminución significativa del mismo (hipopnea) a pesar del esfuerzo inspiratorio que tiene lugar durante el sueño, constituyen parte del síndrome de apnea-hipopnea obstructiva del sueño (SAHS).

El SAHS afecta del 1 al 4\% de la población y aumenta la morbimortalidad de quienes lo padecen, por asociarse a enfermedades cardiovasculares, accidentes de tránsito y dificultades laborales.

El médico general debe estar preparado para su detección pues la falla en su reconocimiento incrementa la mortalidad y deteriora la calidad de vida de estos pacientes.

El SAHS afecta del 2 al $4 \%$ de los varones de edad media y del 1 al $2 \%$ de las mujeres de esta edad (población activa laboralmente). Casi el $80 \%$ de los pacientes son varones. La mayoría de estos pacientes son obesos, presentando en más de la mitad de los casos un índice de masa corporal (en inglés body mass index o BMI) mayor a 30. Las apneas-hipopneas del sueño, pueden responder a un mecanismo obstructivo, central o mixto.El $90 \%$ de las apneas del sueño son de tipo obstructivo.

Los factores asociados a la disminución de la permeabilidad de la vía aérea superior (VAS) durante el sueño que se vinculan al SAHS de tipo obstructivos son: la obesidad, el sexo masculino, el consumo de alcohol y/o sedantes, la enfermedad de Marfan, la acromegalia y el hipotiroidismo. Los más importantes se han discutido en el enfoque del paciente roncador. En forma infrecuente, puede observarse SAHS en pacientes no obesos con alteraciones craneofaciales (evidentes o no), hipertrofia amigdalina severa, antecedentes familiares (el componente genético es discutido) u otros factores que condicionen la reducción del calibre de las VAS en uno o más puntos. El SAHS de tipo obstructivo, el más frecuente, tiene como característica el aumento del esfuerzo respiratorio para reestablecer o mantener el flujo aéreo. En las apneas-hipopneas de mecanismo central existe también, por definición, cese o disminución del flujo aéreo, pero el esfuerzo respiratorio está abolido o reducido, no habiendo obstrucción de las VAS. En las apneas-hipopneas de mecanismo mixto hay obstrucción en las VAS y un esfuerzo respiratorio inadecuado.

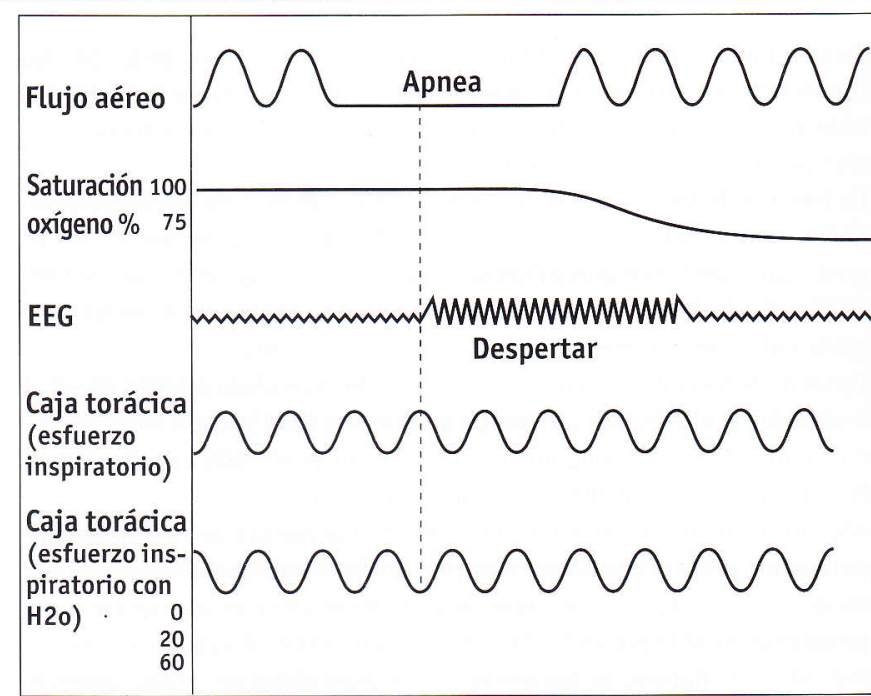

Nótese el aumento del esfuerzo respiratorio que coincide con el despertar, aun en ausencia de desaturación significativa

Como puede verse en la figura 1 , el decremento crítico durante el sueño del calibre de la VAS, en uno o más puntos, genera un mecanismo de despertar, que logra aumentar el tono de los músculos dilatadores de la faringe y devolver así la permeabilidad a la vía aérea. Este mecanismo se acompaña de un aumento del tono simpático (aún en ausencia de hipoxemia) y puede tener lugar cientos de veces durante la noche. Esta activación simpática repetida, sería una de las causas del mayor riesgo de enfermedades cardiovasculares en pacientes con SAHS.

Cada vez que tienen lugar estos fenómenos, en general durante el sueño REM, el paciente vuelve a etapas superficiales del sueño (no suele despertar por completo) y, al ocurrir cientos de veces por noche, provoca una severa disrupción del sueño. Este trastorno es la principal causa de somnolencia diurna y de sueño no reconfortante, lo que tiene un claro impacto negativo en la calidad de vida de los pacientes con SAHS.

Más de la mitad de los pacientes con SAHS son varones, obesos e hipertenSos. El $95 \%$ ronca y el $90 \%$ presentan una excesiva somnolencia diurna. Casi la mitad refiere tener un sueño no reconfortante, cefaleas matinales y episodios de ahogo nocturno. Con mucha frecuencia se observa disfunción cognitiva.

El SAHS se asocia con aumento del riesgo de hipertensión arterial diurna, accidentes cerebrovasculares, infarto agudo de miocardio, insuficiencia cardíaca e hipertensión pulmonar. Estos pacientes suelen tener o han tenido problemas laborales y/o accidentes de tránsito en relación a la somnolencia diurna.

Un estudio poblacional de buen diseño ha reconocido al SAHS como un factor de riesgo independiente para hipertensión arterial diurna, si bien la causalidad es terreno complejo y discutido. En el examen físico se recalca nuevamente el control de peso y la tensión arterial, la inspección del cuello y la VAS. Deben buscarse estigmas de insuficiencia cardíaca izquierda y/o derecha y de enfermedad vascular periférica. El examen pulmonar detallado y , si la historia lo avala , a la sospecha de una enfermedad pulmonar obstructiva crónica, asociación no infrecuente.

\section{Estudios en el SAHS}

La evidencia indica que son dos los grupos de pacientes en los que se debe sospechar e investigar las apneas del sueño:

1. Los roncadores habituales con somnolencia diurna excesiva no explicada por otra razón evidente (ej. consumo de sedantes, alcohol, hipotiroidismo o deprivación de sueño).

2. Los roncadores habituales en quienes fueron observados ceses de la respiración (sobre todo si estos pacientes son obesos e hipertensos).

Los diagnósticos diferenciales a tener en cuenta son el abuso de sedantes y/o alcohol, la deprivación de sueño, las enfermedades psiquiátricas, el mioclono nocturno, la hipersomnolencia idiopática y la narcolepsia.

El test de referencia ("gold standart") para el diagnóstico de las apneas 
del sueño es la polisomnografía. Este estudio consiste en el estudio de una noche de sueño mientras se monitorean los movimientos toracoabdominales, el flujo respiratorio, el electroencéfalograma, la saturación arterial de oxígeno y el electrocardiograma.

Este estudio sirve para el diagnóstico y para el monitoreo del tratamiento. Algunos autores plantean, la realización de monitoreos de menor cantidad de horas de duración, con el objetivo de reducir los costos y de ganar practicidad para el paciente.

Siendo la polisomnografía un estudio costoso y de cierta complejidad, muchos han tratado de establecer un rol para la saturación arterial de oxígeno aislada durante el sueño como método de rastreo en quienes se sospecha SAHS. Si bien es de fácil realización, la sensibilidad de este método es sólo del $60 \%$ (alta tasa de falsos positivos). Es importante remarcar que la somnolencia excesiva de los pacientes con SAHS tiene más relación con el número de despertares, que con la hipoxemia.

\section{Tratamientos}

El enfoque para el tratamiento debe ser multidisciplinario, contemplándose las siguientes medidas:

1. Las medidas generales (disminución de peso, suspensión de alcohol, sedantes y tabaquismo, decúbito lateral al dormir) son similares a las comentadas para el paciente roncador.

2. Presión positiva continua en la vía aérea (más conocida por su sigla inglesa (PAP). Es el tratamiento de elección en los pacientes con SAHS. Ha demostrado disminuir la mortalidad y mejorar la calidad de vida de estos pacientes. Consiste en la utilización de aparatos que gerıeran presión positiva en la vía aérea, adaptándose a la misma por medio de máscaras nasales o nasobucales y evitando así, el colapso en la VAS. Se utiliza durante las horas de sueño. El nivel de presión necesario se establece durante la polisomnografía. Reduce la somnolencia diurna y mejora el desempeño cognitivo de los pacientes con SAHS. Reducen notablemente el número de despertares y de desaturaciones. Disminuyen la incidencia de enfermedad cardiovascular y la mortalidad en estos pacientes. Es un a estrategia costoefectiva en los pacientes con SAHS y es bien tolerada por el $90 \%$ de los pacientes. Es importante destacar que es un tratamiento que es efectivo mientras se utiliza; de ser abandonado la sintomatología recurre a la brevedad. Los roncadores asintomáticos tienen una baja tolerancia al CPAP, esta gran diferencia en la aceptación se origina en la notable mejoría que los pacientes con SAHS experimentan con este tratamiento.

3. Cirugía: no es primera linea de tratamiento, excepto en aquellos con alteraciones del macizo facial o hipertrofia amigdalina severa. También puede considerarse en quienes no toleran el CPAP y tienen cuadros moderados a severos. La cirugía más estudiada en SAHS es la uvulopalatofaringoplastia (UPPP). Su éxito oscila entre el 10 y el $64 \%$. Sus principales problemas son que su beneficio podría diluirse con el tiempo y que no ha demostrado disminuir la mortalidad. Cuando se considera el tratamiento quirúrgico, deben realizarse estudios de imágenes de la VAS (tomográficos o por resonancia) con el fin de evaluar anatómicamente la región y, eventualmente, comprobar si existe algún sitio de angostamiento. Así y todo, ningún método por imágenes predice el éxito de la cirugía, debiéndose probablemente esto a que la obstrucción de la VAS es un fenómeno dinámico que tiene lugar durante el sueño, mientras que estos estudios son estáticos y evalúan al paciente despierto.

4. Aparatos de adelantamiento mandibular o aplicadores orales para aumentar la permeabilidad de la VAS. Pueden ser útiles en pacientes con SAHS leves, 0 en aquellos con cuadros moderados que no han tolerado el CPAP.

5. Oxigenoterapia nocturna: disminuye el número de desaturaciones pero no afecta el de apneas ni la somnolencia diurna. Puede ser una opción cuando no se tolera el CPAP y se desea reducir la duración de los episodios de hipoxemia.

6. Drogas: se han experimentado distintas drogas sin éxito. La única que mejora el cuadro de SAHS es la levotiroxina en pacientes con hipotiroidismo.

\section{¿Cuándo inteconsultar?}

Debe consultarse al neumonólogo o al especialista en trastornos de sueño cuando se ha detectado por polisomnografía SAHS. Recordar que la polisomnografía es un estudio costoso en nuestro medio y que debe ser indicado por el médico general sólo en el grupo de pacientes con alta probabilidad de padecer SAHS. Se sugiere pedir opinión experimentada en los casos con sospecha moderada de diagnóstico de SAHS. La determinación del tratamiento y la forma de seguimiento debe ser establecida por el especialista.

En los pacientes obesos se sugiere el abordaje junto al apoyo del personal del equipo de salud con experiencia en consejo nutricional o especialistas en nutrición.

Dr. Martín Stryjewski Clínica Médica. CEMIC

\section{Bibliografía recomendada}

1. Hoffstein V., MD, PhD, Snoring, Chest 1996; 109: 201-222. (Revisión completa sobre fisiopatología, asociación probable del ronquido con enfermedades vasculares y tratamiento).

2. Jennum P., MD, Hein H.,MD, Saudicani P.,MD, Gyntelberg F.,MD, Risk of Ischemic Heart Disease in Self-reported Snorers, Chest 1995; 108: 138-142. (Estudio de 2937 hombres entre 54 a 74 años sobre asociación entre ronquido y enfermedad cardiovascular).

3. Braver H. M., MD, Block J., MD, FCCP, Perri M.G., PhD, Treatment for Snoring, Chest 1995; 107: 1283-1288. (Estudio sobre tratamiento combinado en pacientes roncadores).

4. Strollo P.J., MD, Roger R.M., MD, Obstructive Sleep Apnea, New England J. Med. 1996; 334: 99-104. (Revisión práctica y conceptual sobre apneas del sueño). 5. Douglas N.J., MD, The Sleep Apnoea-Hypopnoea Syndrome and Snoring, BMJ 1993; 306: 1057-1060. (Breve y excelente enfoque de revisión clínico sobre apneas del sueño).

6. Douglas N.J.,MD, Polo 0.,MD, Pathogenesis of obstructive sleep apnoea-hypopnoea syndrome, Lancet 1994; 344: 653-655. (Buena revisión fisipatológica sobre apneas obstructivas del sueño).

7. Polo 0.,MD, Berthon -Jones M.,PhD, Douglas N.J.,FRCPE, Sullivan C.E.,FRACP, Management of obstructive sleep apnoea-hypopnoea syndrome, Lancet 1994; 344: 656-660. (Enfoque de tratamiento orientado hacia CPAP).

8. Hoffstein V., MD, Blood pressure, snoring, obesity, and nocturnal hypoxaemia, Lancet 1994; 344: 643-645.(Trabajo que estudia la relación entre hipertensión arterial y ronquido, con y sin apneas del sueño).

9. Hla K. M., MD, MHS, Young T., PhD, Bidwell T., MS, Palta M., PhD, Skatrud J.B., MD, Dempsey J., PhD, Sleep Apnea and Hypertension, Ann Intern Med 1994; 120: 382-388. (Estudio poblacional que muestra al SAHS como factor de riesgo independiente para hipertensión arterial).

10. Mendelson W.B., MD, The Relationship of Sleepiness and Blood Pressure to Respiratory Variables in Obstructive Sleep Apnea., Chest 1995; 108: 966-972.

11. Epstein L.J., MD, FCCP, Dorlac G.R., MD, Cost-effectiveness Analysis of Nocturnal Oxymetry as a Method of Screening for Sleep Apnea-Hypopnea Syndrome., Chest 1998; 113: 97-103.

12. Hudgel D.W., MD, FCCP, Treatment of Obstructive Sleep Apnea, Chest 1996; 109: 1346-1358. (Revisión completa sobre tratamiento de SAHS).

13. Stradling J,FRCP, Sleep apnoea and the misuse of evidence-based medicine. (Punto de vista sobre el uso de CPAP basado en evidencia en SAHS).

14. Marklund M., DDS, Franklin K.A., MD, PhD, FCCP, Sahlin C., RTA, Lundgren R, MD, PhD, FCCP, The Effect of a Mandibular Advancement Device on Apneas and Sleep in Patients with Obstructive Sleep Apnea, Chest 1998; 113: 707-713.

15. Phillips B., MD, FCCP., Collop N., MD, FCCP, Strollo P., MD, Controversies in Sleep Disorders, Chest 1996, 110: 1597-1602.(Planteo de controversias entre especialistas, respecto a las definiciones en enfermedades del sueño). 